\title{
Dietary $\gamma$-Aminobutyric Acid Shortens the Life Span of Stroke-Prone Spontaneously Hypertensive Rats
}

\author{
Nakamichi Watanabe, Naomi Washio \\ Department of Food Science and Nutrition, Showa Women's University, Tokyo, Japan. \\ Email: nakamich@swu.ac.jp \\ Received September 15 $5^{\text {th }}, 2010$; revised February 12 $2^{\text {th }}, 2011$; accepted March $1^{\text {st }}, 2011$.
}

\begin{abstract}
Dietary $\gamma$-amino butyric acid (GABA) has been suggested to decrease systolic blood pressure. This study aimed to examine the effects of dietary GABA on the life span of stroke-prone spontaneously hypertensive rats (SHRSPS). In this study, life span was determined for SHRSPs provided $1 \% \mathrm{NaCl}$ solution or $0.01 \%$ GABA in $1 \% \mathrm{NaCl}$ solution as drinking water. The life span of the GABA-fed group (76.3 \pm 1.65 days) was significantly shorter than that of the control group ( $81.6 \pm 0.88$ days). The results of this study may not be applicable to humans. Future studies will be necessary to elucidate the mechanism underlying this phenomenon.
\end{abstract}

Keywords: $\gamma$-Amino Butyric Acid, Stroke-Prone Spontaneously Hypertensive Rat, Life Span

\section{Introduction}

$\gamma$-Aminobutyric acid (GABA) is an amino acids, found in unpolished rice, fermented food such as tempe, and vegetables. In humans, it is well recognized as an inhibitory transmitter. Moreover GABA is present in the peripheral organs such as the intestinal tract; [1] however, it has also been reported that dietary GABA reduces increased systolic blood pressure [1], improves discrimination learning [2], and alleviates stress [3]. In particular, many studies of the effect of GABA on blood pressure have been performed, and dietary GABA has been approved as a "Food for Specific Health Use" in Japan. The antihypertensive effect of GABA is thought to be caused by the suppression of noradrenalin secretion from the sympathetic nerve mediated by $\mathrm{GABA}_{\mathrm{B}}$ receptor, and the inhibition of angiotensin-converting enzyme II [4]. Therefore, dietary GABA may prevent cardiovascular disease such as cerebral stroke, but no study has examined the effect of GABA on stroke onset yet. The present study aimed to examine the effect of dietary GABA on the life span of stroke-prone spontaneously hypertensive rats (SHRSPs).

\section{Materials and Methods}

\subsection{Animals and Diets}

SHRSPs aged 3 weeks were purchased from Japan SLC
Inc. (Shizuoka, Japan). The animals were housed individually in stainless steel cages in a room with controlled lighting (lights on: 0800 - 2000 hours) and a constant temperature $\left(20-25^{\circ} \mathrm{C}\right)$. They were fed a commercial nonpurified chow diet (CRF-1; CLEA Japan Co. Ltd.; Tokyo, Japan) and distilled water for 1 week. They were then divided into 2 experimental groups of 5 rats each. The food remained the same, but the water was replaced with $1 \% \mathrm{NaCl}$ solution in 1 group and with $0.01 \%$ GABA solution in $1 \% \mathrm{NaCl}$ in the other group. GABA (special-grade reagent) was purchased from Wako pure chemical industries, Ltd. (Osaka, Japan). The concentration of the GABA solution was the same as that in Gabaron Tea [5], which is a commercially available Japanese tea rich in GABA. The rats were given access to the food and water ad libitum. Their body weight, food intake, and water intake were measured once every week during the experimental period. The life span and the period from the onset to the death of the animals were recorded. If an animal died, necropsy was performed to confirm stroke. All animal studies were performed according to the approved animal research protocol of Showa Women's University.

\subsection{Statistical Analysis}

Data are represented as means \pm standard error. The dif- 
ferences between the experimental groups were evaluated by Student's t test. $P<0.05$ was considered statistically significant.

\section{Results and Discussion}

Until the onset of stroke, the body weights of all animals increased well, and no significant differences were observed between the 2 groups (Figure 1). Food and water intake also did not differ (data not shown).

As shown in Figure 2, the life span of the GABA group (76.3 \pm 1.65 days) was significantly shorter than that of the control group ( $81.6 \pm 0.88$ days). As shown in Figure 3, the periods from stroke onset to death in the GABA group ( $3.8 \pm 1.7$ days $)$ was not significantly different from that in the control group ( $4.5 \pm 2.3$ days).

Although several animal studies, including those on

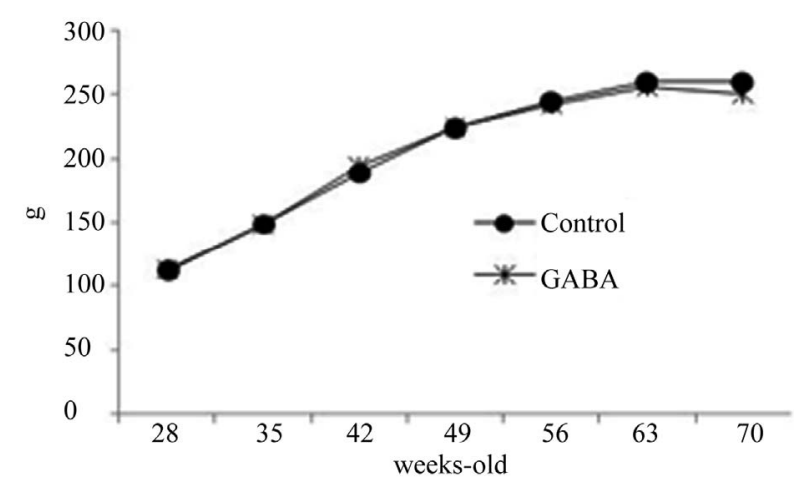

Figure 1. Changes of body weight of SHRSPs.

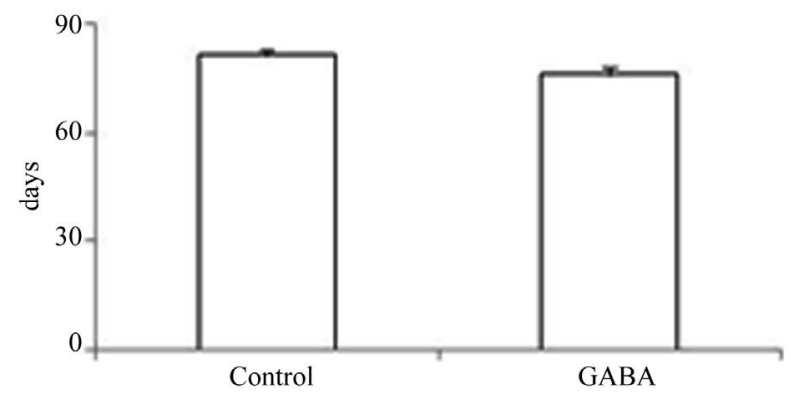

Figure 2. Life spans of SHRSPs.

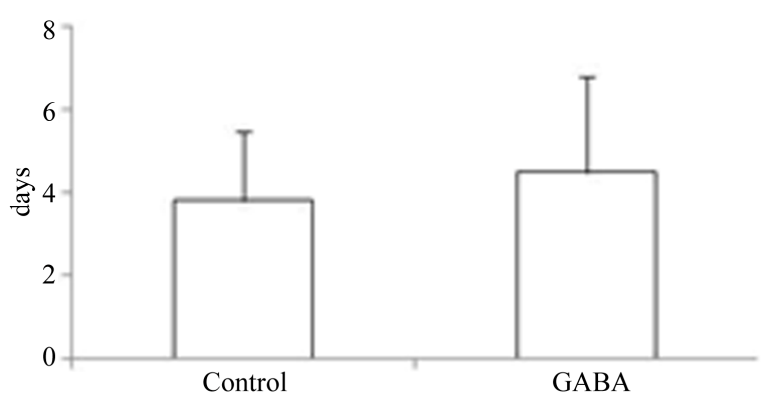

Figure 3. Periods from stroke onset to death of SHRSPs. spontaneously hypertensive rat (SHRs), have shown that dietary GABA decreases systolic blood pressure [4], we found that the life span of SHRSPs fed GABA was less than that of the control rats. In our study, we did not measure the animals' blood pressure, since we wanted to prevent excess stress that might have increased blood pressure and promoted stroke onset. Therefore, we could not confirm the antihypertensive effects of GABA in the SHRSPs.

The results of this animal study might not be applicable to humans because the brain uptake of GABA is suggested to be a characteristic of SHRs [6,7]. Generally, it has been reported that polar molecules such as dietary GABA cannot cross the brain because of the blood-brain barrier (BBB). However, the volume of ${ }^{14} \mathrm{C}-\mathrm{GABA}$ distribution in the cerebrospinal fluid and brain regions was significantly greater in SHRs than in Wistar Kyoto (WKY) rats. Moreover, a study on BBB integrity showed that the paracellular permeability of the brain capillaries was higher in SHRs than in WKY rats [8].

Another difference between human and SHRSP is the excessively high absorption of phytosterol induced by mutation of ATP-binding cassette transporter G5 [9].

Therefore, if SHRSP was used in a nutritional experiment, specific consideration might be needed.

Although the abnormality of the BBB in SHRSPs has not been examined in this respect, a BBB disorder may be related to the short life span observed for GABA-fed SHRSPs in this study. The underlying mechanism should be elucidated in future studies.

\section{Conclusions}

We observed the life span of SHRSP fed GABA because dietary GABA reduces systolic blood pressure. Unexpectedly, dietary GABA decreased the life span of SHRSP. The results of this animal study might not be applicable to humans because the brain uptake of GABA is a characteristic of SHRs. The underlying mechanism for this phenomenon will be elucidated in future studies.

\section{REFERENCES}

[1] S. Vemulapalli and M. Barletta, "The Role of the Sympathetic Nervous System in the Cardiovascular Effects of Systemically Administrated $\gamma$-Aminobutyric Acid," Archives Internationales de Pharmacodynamie et de Thérapie, Vol. 267, No. 1, 1984, pp. 46-58.

[2] K. Ishikawa and S. Saito, "Effect of Intraventricular $\gamma$-Aminobutyric Acid (GABA) on Discrimination Learning in Rats," Psychopharmacology, Vol. 56, No. 2, 1978, pp. 127-132. doi:10.1007/BF00431837

[3] H. Nakamura, T. Takishima, T. Kometani and H. Yokogoshi, "Psychological Stress-Reducing Effect of Chocolate Enriched with $\gamma$-Aminobutyric Acid (GABA) 
in Humans: Assessment of Stress Using Heart Rate Variability and Salivary Chromogranin A," International Journal of Food Sciences and Nutrition, Vol. 22, Supplement 5, 2009, pp. 1-8.

[4] H. Kazuhiro, K. Masayuki and K. Kazuo, "Mechanism Underlying $\gamma$-Aminobutyric Acid-Induced Antihypertensive Effect in Spontaneously Hypertensive Rats," European Journal of Pharmacology, Vol. 438, No. 1-2, 2002, pp. 107-113.

[5] K. Hayakawa, M. Kimura, K. Kasaha, K. Matsumoto, H. Sansawa and Y. Yamori, "Effects of a $\gamma$-Aminobutyric Acid-Enriched Dairy Product on the Blood Pressure of Spontaneously Hypertensive and Normotensive Wistar-Kyoto Rats," British Journal of Nutrition, Vol. 92, No. 3, 2004, pp. 411-417. doi:10.1079/BJN20041221

[6] H. Al Sarraf and L. Philip, "Increased Brain Uptake and CSF Clearance of ${ }^{14} \mathrm{C}$-Glutamate in Spontaneously Hypertensive Rats," Brain Research, Vol. 24, No. 2, 2003,

\section{pp. 181-187. doi:10.1016/j.brainres.2003.09.034}

[7] M. Al Awadi, A. Pavlik and H. Al Sarraf, "Increased Brain Uptake and Brain Efflux Transport of ${ }^{14} \mathrm{C}-\mathrm{GABA}$ in Spontaneously Hypertensive Rats," Life Sciences, Vol. 79, No. 9, 2006, pp. 847-853. doi:10.1016/j.lfs.2006.02.039

[8] V. Carelli, F. Liberatore, L. Scipione, G. Giorgioni, A. D. Stefano and M. Impicciatore, "Synthesis and Biological Evaluation of GABA Derivatives Able to Cross the Blood-Brain Barrier in Rats," Bioorganic \& Medicinal Chemistry Letters, Vol. 13, No. 21, 2003, pp. 3765-3769. doi:10.1016/j.bmcl.2003.07.004

[9] K. A. Scoggan, H. Gruber and K. Lariviere, "A Missense Mutation in the Abcg5 Gene Causes Phytosterolemia in SHR, Stroke-Prone SHR, and WKY Rats," Journal of Lipid Research, Vol. 44, No. 5, 2003, pp. 911-916. doi:10.1194/jlr.M200438-JLR200 